Canadian Science Publishing

Canadian Journal of Physics

Revue canadienne de physique

\title{
Extended Analysis of Xe VII and Xe VIII
}

\begin{tabular}{|r|l|}
\hline Journal: & Canadian Journal of Physics \\
\hline Manuscript ID & Cjp-2016-0728.R1 \\
\hline Manuscript Type: & Review \\
\hline Date Submitted by the Author: & 05-Dec-2016 \\
\hline Complete List of Authors: & $\begin{array}{l}\text { Raineri, Mónica; Centro de Investigaciones Opticas, } \\
\text { Gallardo, Mario; Centro de Investigaciones Opticas } \\
\text { Reyna Almandos, Jorge; Centro de Investigaciones Opticas } \\
\text { Pagan, Cesar; School of Electrical and Computer Engineering, University of } \\
\text { Campinas (UNICAMP), 13083-852, Campinas, SP, Brazil } \\
\text { Sarmiento, Rafael; Grupo de Espectroscopía Optica de Emisión y Láser, } \\
\text { GEOEL, Universidad del Atlántico, AA. 1890, Barranquilla, Colombia }\end{array}$ \\
\hline Keyword: & atomic, spectroscopy, xenon, spectral, analysis \\
\hline \multicolumn{2}{|c}{} \\
\hline
\end{tabular}

SCHOLARONE $^{m}$

Manuscripts 


\title{
Extended Analysis of Xe VII and Xe VIII ${ }^{1}$
}

\section{Raineri, M. Gallardo, J. Reyna Almandos, C. J. B. Pagan and R. Sarmiento}

\begin{abstract}
A pulsed discharge light source to study the six and seven times ionized xenon spectra in the 419-4642 \& region was used. A set of 40 transitions of Xe VII and 25 transitions of Xe VIII were classified for the first time. We revised the values for the previously known energy levels and extended the analysis for Xe VII to 10 new energy levels belonging to $5 \mathrm{~s} 6 \mathrm{~d}, 5 \mathrm{~s} 7 \mathrm{~s}$ and $5 \mathrm{~s} 7 \mathrm{p}, 4 \mathrm{~d}^{9} 5 \mathrm{~s}^{2} 5 \mathrm{p}$ even and odd configurations, respectively. 7 new energy levels of the core excited configuration $4 \mathrm{~d}^{9} 5 \mathrm{~s} 5 \mathrm{~d}$ of Xe VIII are presented. For the prediction of the atomic parameters, energy levels and transition, relativistic Hartree-Fock calculations were used.
\end{abstract}

PACS: 32,30 Jc. $52,80 \mathrm{Yr}$

Keywords: Atomic spectra, Energy levels, Capillary-pulsed discharge, Relativistic Hartree-Fock calculations

\section{Introduction}

The spectral analysis of several ions of xenon has implications for astronomy and laser studies. Various atomic parameters such as energy levels, oscillator strengths, transition probabilities and radiative lifetimes have many important astrophysical applications. Transition probabilities are needed for calculating the energy transport through the star in model atmospheres [1] and for direct analysis of stellar chemical compositions [2]. Xenon is a very rare element in the cosmos, observed in chemically peculiar stars [3] and in planetary nebulae [4]. Xe VI and Xe VII lines were observed in the ultraviolet spectrum of the hot DO-type white dwarf RE 0503-289 [5-6], in the first detection of xenon in this kind of star.

$\mathrm{Xe}$ VII belongs to the $\mathrm{Cd}$ isoelectronic sequence and has the ground configuration $5 \mathrm{~s}^{2}$. In the studies related with the stimulated emission of xenon, the understanding of the population mechanisms that affect the Xe VII laser action requires knowledge of the spectral analysis corresponding to this ion. Results for ions in the Cdlike isoelectronic sequence were published [7-17] and previous works about cadmiumlike xenon were carried out by different groups of researchers [18-23], who used various experimental methods such as beam-foil spectroscopy, spark-light sources, laserproduced plasma, theta-pinches, etc. Larsson et al. [24] obtained energy levels of the $5 \mathrm{~s} 6 \mathrm{~s}, 5 \mathrm{~s} 5 \mathrm{~d}$ and $5 \mathrm{p}^{2}$, and $5 \mathrm{~s} 4 \mathrm{f}$ configurations by collision-based spectroscopy. Using the

${ }^{1}$ M. Raineri, M. Gallardo and J. Reyna Almandos. Centro de Investigaciones Ópticas (CIOp), CC 3, 1897, Gonnet, La Plata, Argentina

C. J. B. Pagan. School of Electrical and Computer Engineering, University of Campinas (UNICAMP), 13083-852, Campinas, SP, Brazil

R. Sarmiento. Grupo de Espectroscopía Óptica de Emisión y Láser, GEOEL, Universidad del Atlántico, AA. 1890, Barranquilla, Colombia

Corresponding author: M.Raineri (e-mail: monicar@ciop.unlp.edu.ar) 
same kind of excitation, Wang et al.[25] extended Larsson's work to include the $5 \mathrm{~s}(6 \mathrm{p}$ $+7 p)$ and $5 s(6 d+7 s)$ configurations. The study of the $5 p 5 d$ configuration by Cavalcanti et al using a theta-pinch as light source [26], by Gallardo et al, reporting fourteen new energy levels and one hundred and ten new lines using a pulsed discharge [27], was made. After that, Churilov and Joshi [28] used two different light sources to study this ion and reported thirty nine new energy level values.

Seven times ionized xenon, Xe VIII, belongs to the Ag I isoelectronic sequence and its ground state is $5 \mathrm{~s}^{2} \mathrm{~S}_{1 / 2}$. The spectrum of this ion has been investigated by many groups using different spectral sources, e.g., by beam foil spectroscopy [29], by use of an inductance-triggered spark as light source [30-32] by CBS [33,34] and by a discharge tube [35,36]. Thereby, the configurations $5 \mathrm{~s}-9 \mathrm{~s}, 5 \mathrm{p}-9 \mathrm{p}, 5 \mathrm{~d}-9 \mathrm{~d}, 4 \mathrm{f}-9 \mathrm{f}, 5 \mathrm{~g}-9 \mathrm{~g}$, $6 \mathrm{~h}-9 \mathrm{~h}, 7 \mathrm{i}-10 \mathrm{i}, 8 \mathrm{k}-10 \mathrm{k}, 91$ have been established. Also some levels of core excited $4 d^{9} 5 s 5 p, 4 d^{9} 5 s 6 p$ and $4 d^{9} 5 s 5 f$ configurations have been determined. In addition to these analyses some authors [29,30,34] have also established quantum defects of Rydberg levels important to predict energies of unknown levels and to derive ionization energies and core polarizabilities. In the work of M. Gallardo et al [37], using a capillary light source, twenty-eight energy levels belonging to the series Rydberg configurations were adjusted and 38 new spectral lines were classified.

The experimental results on energy levels and transitions of Xe VII and Xe VIII spectrum were critically compiled by Saloman [38]. Lifetimes and transition probabilities on these ions were reported by Biemont et al [39].

In order to continue the study of the six and seven times ionized xenon spectrum, a new spectral analysis of these ions is presented in this work. This study is a continuation of our research on different xenon ion spectra using gases pulsed electrical discharges [40-43] (and references therein). We used experimental data of the xenon spectrum covering the wavelength range $230-7000 \AA$ for the UV-Visible region. A set of 40 transitions of Xe VII and 25 transitions of Xe VIII were classified for the first time. We revised the values for the previously known energy levels and extended the analysis for Xe VII to 10 new energy levels belonging to $5 \mathrm{~s} 6 \mathrm{~d}, 5 \mathrm{~s} 7 \mathrm{~s}$ and $5 \mathrm{~s} 7 \mathrm{p} 4 \mathrm{~d}^{9} 5 \mathrm{~s}^{2} 5 \mathrm{p}$ even and odd configurations, respectively. 7 new energy levels of the $4 d^{9} 5 \mathrm{~s} 5 d$ core excited configuration of Xe VIII are presented. Theoretical predictions of the configuration structure were obtained from the computer code developed by Cowan [44], which provides atomic parameters by means of Hartree-Fock relativistic (HFR) approximation. The energy matrix was calculated using energy parameters adjusted to fit the experimental energy levels.

\section{Experiment}

The light source used in this work was a capillary discharge experimental set built at CIOp [40], consisting of a Pyrex tube of $100 \mathrm{~cm}$ long and inner diameter of 0.5 $\mathrm{cm}$, in which the gas excitation was produced by discharging a bank of low-inductance capacitors ranging from 20 to $280 \mathrm{nF}$, charged with voltages up to $20 \mathrm{kV}$. In the wavelength range below $2000 \AA$, light radiation emitted axially was analyzed using a 3 $\mathrm{m}$ normal incidence vacuum spectrograph with a concave diffraction grating of 1200

lines $\mathrm{mm}^{-1}$. The plate factor in the first order was $2.77 \AA \mathrm{mm}^{-1}$. C, N, O and known 
lines of xenon served as internal wavelength standards. The wavelength range above $2000 \AA$ was recorded on a $3.4 \mathrm{~m}$ Ebert plane-grating spectrograph, with a diffraction grating of 600 lines $\mathrm{mm}^{-1}$. The plate factor was $5.0 \AA \mathrm{mm}^{-1}$ in the first order. The spectra were recorded in the first, second, and third diffraction orders. $\mathrm{Th}^{232}$ lines from an electrodeless discharge were superimposed on the spectrograms and served as reference [45]. The positions of the observed Xe VII and Xe VIII spectral lines were determined by means of a rotating prism photoelectric semiautomatic Grant comparator. For sharp lines, the settings were reproducible within $\pm 1 \mu \mathrm{m}$. The uncertainty in the determination of the wavelength of unperturbed lines was estimated to be $\pm 0.02 \AA$ for the VUV region and $\pm 0.01 \AA$ for the visible region. The intensity figures are visual estimates of photographic density, and are on a uniform scale only within limited wavelength ranges. To distinguish among different states of ionization, we studied the behavior of the spectral line intensity as a function of pressure.

\section{Results and discussions}

For the theoretical predictions and interpretation of these spectra Cowan's code [44] was used. These theoretical values were obtained by diagonalizing the energy matrices with adjusted radial parameters that minimized the discrepancies between the eigenvalues and the available experimental energies. In this work we present a revised and extended analysis for the $5 \mathrm{~s} 6 \mathrm{~d}, 5 \mathrm{~s} 7 \mathrm{~s}$ and $5 \mathrm{~s} 7 \mathrm{p}, 4 \mathrm{~d}^{9} 5 \mathrm{~s}^{2} 5 \mathrm{p}$ even and odd configurations of Xe VII and for the $4 \mathrm{~d}^{9} 5 \mathrm{~s} 5 \mathrm{~d}$ configuration of Xe VIII. The configuration sets used were $5 \mathrm{~s}^{2}, 5 \mathrm{p}^{2}, 5 \mathrm{~s}(5 \mathrm{~d}+6 \mathrm{~d}+7 \mathrm{~d}+8 \mathrm{~d}), 5 \mathrm{~s}(6 \mathrm{~s}+7 \mathrm{~s}), 5 \mathrm{p}(4 \mathrm{f}+5 \mathrm{f}), 5 \mathrm{p} 6 \mathrm{p}$, $4 \mathrm{f}^{2}, 5 \mathrm{~d}^{2}, 4 \mathrm{~d}^{9} 5 \mathrm{~s}^{2} 5 \mathrm{~d}, 4 \mathrm{~d}^{9} 5 \mathrm{~s}^{2} 6 \mathrm{~s}$ and $5 \mathrm{~s}(5 \mathrm{p}+6 \mathrm{p}+7 \mathrm{p}), 5 \mathrm{~s}(4 \mathrm{f}+5 \mathrm{f}), 6 \mathrm{~s} 4 \mathrm{f}, 5 \mathrm{p}(5 \mathrm{~d}+6 \mathrm{~s}), 4 \mathrm{f} 5 \mathrm{~d}$, $4 d^{9} 5 s^{2} 5 \mathrm{p}, 4 \mathrm{~d}^{9} 5 \mathrm{~s}^{2} 4 \mathrm{f}$ for the Xe VII even and odd parities respectively, and $5 \mathrm{~s}-10 \mathrm{~s}, 5 \mathrm{~d}-$ $10 \mathrm{~d}, 5 \mathrm{~g}-10 \mathrm{~g}, 7 \mathrm{i}-10 \mathrm{i}, 4 \mathrm{~d}^{9} 5 \mathrm{~s}^{2}, 4 \mathrm{~d}^{9} 5 \mathrm{~s} 5 \mathrm{~d}, 4 \mathrm{~d}^{9} 5 \mathrm{p}^{2}$ and $5 \mathrm{p}-10 \mathrm{p}, 4 \mathrm{f}-10 \mathrm{f}, 6 \mathrm{~h}-10 \mathrm{~h}, 8 \mathrm{k}-10 \mathrm{k}$, $4 d^{9} 5 s 5 p, 4 d^{9} 4 f 5 s, 4 d^{9} 5 p 5 d$ for Xe VIII even and odd parities. Table 1 shows the new and adjusted energy level values for these ions. These values were determined in an iterative procedure where the wavenumbers of the observed lines were weighted according to their estimated uncertainties. All level designations in the table are in LS notation. We also present the percentage composition of the levels. The uncertainties of the adjusted experimental energy level values were generally less than $2 \mathrm{~cm}^{-1}$. Table 2 shows the new classified lines for Xe VII and Xe VIII. The values of $\sigma_{\text {obs }}$ and $\sigma_{\text {cal }}$ in this table are the observed and calculated Ritz wavenumbers respectively. The difference between these values is in accordance with the uncertainty in the determination of the wavelength. In our analysis we also calculated the oscillator strengths gf and transition probabilities gA (the latter shown in Table 2), in order to compare with the experimental intensity of the new observed lines.

We adjusted the energy level values reported by Wang et al. [25] (compiled by Saloman [38]) for ${ }^{3} \mathrm{D}_{1,2,3}$ of $5 \mathrm{~s} 6 \mathrm{~d}$ and ${ }^{3} \mathrm{~S}_{1}$ of $5 \mathrm{~s} 7 \mathrm{~s}$ configurations. We reported new energy level values for ${ }^{1} \mathrm{D}_{2}$ of $5 \mathrm{~s} 6 \mathrm{~d},{ }^{1} \mathrm{~S}_{0}$ of $5 \mathrm{~s} 7 \mathrm{~s},{ }^{1} \mathrm{P}_{1}$ of $5 \mathrm{~s} 7 \mathrm{p}$ configurations and 7 new energy level values for $4 d^{9} 5 s^{2} 5 p$ configuration of Xe VII spectrum.

We presented 7 new energy level values for the $4 d^{9} 5 s 5 d$ core excited configuration of Xe VIII and classified 25 new transitions with the previously known $4 d^{9} 5 s 5 p$ configuration [32]. 
The calculated energy level values were obtained by the least squares fit [44]. In this process, the Slater parameters were fitted to the experimental energy level, which should improve the wave function composition as well as the energy separations between the levels. Our calculations included all the energy levels experimentally known. The least squares calculation results are shown in Tables 3, 4, 5 and 6 for the Xe VII and Xe VIII. In Tables 3 and 4 we show the radial parameters for the even and oddparity configurations of Xe VII. Similar calculations were given by Churilov et al. [28]. In Table 3 the adjusted parameters that where let free are in good agreement with the scaled Hartree-Fock (HF) values. In Table 4 the $G^{3}(4 d, 5 p)$ integral for the $4 d^{9} 5 s^{2} 5 p$ configuration was fixed at $100 \%$ of its HF value because there are values of energy levels that are unknown. We also observed strong configuration interaction between $5 \mathrm{~s} 5 \mathrm{p}-5 \mathrm{p} 5 \mathrm{~d}$ and $5 \mathrm{~s} 5 \mathrm{f}-5 \mathrm{p} 5 \mathrm{~d}$ configurations that were fixed at $75 \%$ and $100 \%$ of their HF values respectively. In Table 5 we show the even parameters for Xe VIII ion, where six of the nine parameters of the $4 d^{9} 5 \mathrm{~s} 5 \mathrm{~d}$ configuration were let free in the calculation, because there are only 7 new experimental energy levels of the 34 levels belonging to this configuration. The other 3 energy parameters $\left.G^{0}(4 d, 5 d), G^{2}(4 d, 5 d), G^{4}(4 d, 5 d)\right)$ were fixed and scaled to $85 \%$ of its HF values in order to reduce the standard deviation. All the configuration-interaction integrals were also scaled at $85 \%$ of their Hartee-Fock (HF) values. Table 6 shows the radial parameter values corresponding to the odd parity configurations. In this table the $\zeta_{4 d}$ and $\mathrm{G}^{3}(4 \mathrm{~d}, 5 \mathrm{p})$ parameters corresponding to the $4 d^{9} 5 s 5 p$ configuration were fixed because there are values of energy levels that are unknown.

In the energy adjustment of the Xe VII the standard deviation was $175 \mathrm{~cm}^{-1}$ and $281 \mathrm{~cm}^{-1}$ for the even and odd parity. For even parity 29 free levels and 20 adjusted parameters were used. In a similar way 47 free levels and 27 adjusted parameters were used for odd parity. The standard deviation was $220 \mathrm{~cm}^{-1}$ and $248 \mathrm{~cm}^{-1}$ for the even and odd parity in Xe VIII. In this case 35 free levels and 28 adjusted parameters for even parity and 40 free levels and 28 adjusted parameters for odd parity were used.

\section{Conclusions}

In this work we studied the Xe VII and Xe VIII spectra covering the wavelength range 230-7000 $\AA$ for the UV-Visible region using a pulsed electrical discharges. A set of 40 transitions of Xe VII and 25 transitions of Xe VIII were classified. We revised the values for the previously known energy levels and extended the analysis for Xe VII to 10 new energy levels and 4 adjusted values belonging to $5 \mathrm{~s} 6 \mathrm{~d}, 5 \mathrm{~s} 7 \mathrm{~s}, 5 \mathrm{~s} 7 \mathrm{p}, 4 \mathrm{~d}^{9} 5 \mathrm{~s}^{2} 5 \mathrm{p}$ configurations. 7 new energy levels of $4 d^{9} 5 \mathrm{~s} 5 \mathrm{~d}$ configuration of Xe VIII were presented. Relativistic Hartree-Fock calculations were used. Least-squares-fitted parametric calculations have been carried out to interpret the observed spectrum.

\section{Acknowledgments}

This research was supported by the Consejo Nacional de Investigaciones Científicas y Técnicas, Argentina, by the Conselho Nacional de Pesquisa (CNPq), Brazil and by the Universidad del Atlántico, Barranquilla, Colombia. The Comision de Investigaciones Cientıficas de la Provıncia de Buenos Aires (CIC), where J. Reyna Almandos and $\mathrm{M}$.Raineri are researchers, is also gratefully acknowledged. 


\section{References}

[1] B. Gustafsson. Phys. Scr. 34, 14 (1991). doi: 10.1088/0031-8949/1991/T34/002

[2] E. Biémont, K. Blagoev, J. Campos, R. Mayo, G. Malcheva, M. Ortíz and P. Quinet. Spectrosc. Relat. Phenom. 27, 144 (2005). doi: 10.1016/j.elspec.2005.01.201

[3] C. R. Cowley, S. Hubrig, P. Palmeri, P. Quinet, É. Biémont, G. M. Wahlgren, O.Schütz and J.F. González. M.N.R.A.S. 405, 1271 (2010). doi: 10.1111/j.13652966.2010.16529.x

[4] M. Otsuka and A.Tajitsu. Ap.J. 778, 146 (2013). doi: 10.1088/0004$637 \mathrm{X} / 778 / 2 / 146$

[5] K. Werner, T. Rauch, E. Ringat and J.W. Kruk. Astrophys. J. Lett. 753, L7 (2012). doi: $10.1088 / 2041-8205 / 753 / 1 / \mathrm{L} 7$

[6] T. Rauch, D. Hoyer, P. Quinet, M. Gallardo and M. Raineri. Astron. Astrophys. 577, A88- 3pp (2015). doi: 10.1051/0004-6361/201526078

[7] A. Tauheed, Y. N. Joshi, E. H. Pinnington. Phys. Scr. 56, 289 (1997). doi: $10.1088 / 0031-8949 / 56 / 3 / 011$

[8] R. Gayasov and Y. N. Joshi. J. Opt. Soc. Am. B 16, 1280 (1999). doi: 10.1364/JOSAB.16.001280

[9] R. Gayasov, Y. N. Joshi and A. N. Ryabtsev. Phys. Scr. 59, 419 (1999). doi: 10.1238/Physica.Regular.059a00419

[10] S. S. Churilov and Y. N. Joshi . Phys. Scr. 62, 282 (2000). doi: 10.1238/Physica.Regular.062a00282

[11] A. Tauheed, Y. N. Joshi and A. F. Zafaran. Phys. Scr. 62, 316 (2000). doi: 10.1238/Physica.Regular.062a00316

[12] A. Tazeen Rana, A. Tauheed and Y.N. Joshi. Phys. Scr. 63, 108 (2001). doi: 10.1238/Physica.Regular.063a00108

[13] Y. N. Joshi, A. N. Ryabtsev and S. S. Churilov. J. Opt. Soc. Am. B 18, 1935 (2001). doi: 10.1364/JOSAB.18.001935

[14] A. N. Ryabtsev, S. S. Churilov and Y. N. Joshi. Phys. Scr. 65, 227 (2002). doi: 10.1238/Physica.Regular.065a00227

[15] S. S. Churilov and Y. N. Joshi. Phys. Scr. 68, 128 (2003). doi: 10.1238/Physica.Regular.068a00128

[16] S. S. Churilov, Y. N. Joshi and A. N. Ryabtsev. Phys. Scr. 71, 43 (2005). doi: $10.1088 / 0031-8949 / 71 / 1 / 007$

[17] S. S. Churilov and Y. N. Joshi. Phys. Scr. 71, 453 (2005). doi: 10.1238/Physica.Regular.071a00453 
[18] B. C. Fawcett, B. B. Jones and R. Wilson. Proc. Phys. Soc. Lond. 78, 1223 (1961). doi: $10.1088 / 0370-1328 / 78 / 6 / 318$

[19] E. J. Knystautas, J. Sugar and J. R. Roberts. J. Opt. Soc. Am. 69, 1726 (1979). doi: 10.1364/JOSA.69.001726

[20] R. Hallin, J. Leavitt, A. Lindgard, P. W. Rathmann, H. Vach and T.Veje. Nucl. Instrum. Meth. 202, 41 (1982). doi: 10.1016/0167-5087(82)90373-8

[21] G. O'Sullivan. J. Phys. B 15, L765 (1982). doi: 10.1088/0022-3700/15/21/002

[22] J. Blackburn, P. K. Carroll, J. Costello and G..O'Sullivan. J. Opt. Soc. Am. 73, 1325 (1983). doi: 10.1364/JOSA.73.001325

[23] V. Kaufman and S. Sugar. J. Opt. Soc. Am. B 4, 1919 (1987). doi: 10.1364/JOSAB.4.001919

[24] M. O. Larsson, M. Gonzalez, R. Hallin, F. Heijkenskjöld, R. Hutton, A. Langereis, B. Nyström, G. O'Sullivan and A. Wännström. Phys. Scr. 51, 69 (1995). doi: $10.1088 / 0031-8949 / 51 / 1 / 011$

[25] M. Wang, R. Arnesen, R. Hallin, F. Heijkenskjöld, M. O. Larsson, A. Wännström, A.G. Trigueiros and A. V. Loginov. J. Opt. Soc. Am. B 14, 3277 (1997). doi: 10.1364/JOSAB.14.003277

[26] G. H. Cavalcanti, F. R. T. Luna, A. G. Trigueiros, F. Bredice, H. Sobral, R. Hutton and M. Wang. J. Opt. Soc. Am. B 14, 2459 (1997). doi: 10.1364/JOSAB.14.002459

[27] M. Gallardo, F. Bredice, M. Raineri, A. G. Trigueiros and J. G. Reyna Almandos. Eur. Phys. J. D 15, 307 (2001). doi: 10.1007/s100530170145

[28] S. S. Churilov and Y. N. Joshi. Phys. Scr. 65, 35 (2002). doi: 10.1238/Physica.Regular.065a00035

[29] S. Bashkin, R. Hallin, J. Leavitt, U. Litzen and D. Walker. Phys Scr. 23, 5 (1981). doi: $10.1088 / 0031-8949 / 23 / 1 / 001$

[30] V. Kaufman and J. Sugar. Phys Scr. 24, 738 (1981). doi: 10.1088/0031$8949 / 24 / 4 / 009$

[31] J.R. Roberts, F.J. Knystautas and J. Sugar. J Opt Soc Am 69, 1620 (1979). doi: 10.1364/JOSA.69.001620

[32] V. Kaufman and J. Sugar. J Opt Soc Am B 1, 38 (1984). doi: 10.1364/JOSAB.1.000038

[33] M.O. Larsson, A.M. Gonzalez, R. Hallin, F. Heijkenskjöld, R. Hutton, A. Langereis, B. Nyström, G. O'Sullivan and A. Wännström. Phys Scr. 51, 69 (1995). doi: 10.1088/0031-8949/51/1/012

[34] M. Wang, A. Arnesen, R. Hallin, F. Heijkenskjöld, A. Langereis, M.O. Larsson, C. Nordling and A. Wännström. J Opt Soc Am B 13, 1650 (1996). doi: 10.1364/JOSAB.13.001650 
[35] M. Gallardo, R. Sarmiento, M. Raineri and J. Reyna Almandos. Proc SPIE 3572, 547 (1999). doi: 10.1117/12.358432

[36] M. Gallardo, F. Bredice, M. Raineri and J. Reyna Almandos. Appl. Opt. 21, 4513 (1989). doi: 10.1364/AO.28.004513

[37]M. Gallardo, M. Raineri, M. Giuliani, C. Lagorio, S. Padilla, R.Sarmiento and J.G. Reyna Almandos. J. Quant. Spectrosc. Radiat.Transfer. 95, 365 (2005).. doi: 10.1016/j.jqsrt.2004.11.004

[38] Saloman E B. J. Phys. Chem. Ref. Data 33, 765 (2004). doi: 10.1063/1.1649348

[39] E. Biémont, V. Clar M, Fivet, H-P Garnir, P. Palmeri, P. Quinet and D. Rostohar. . Eur. Phys. J. D. 44, 23 (2007). doi: 10.1140/epjd/e2007-00161-2

[40] J. Reyna Almandos, F. Bredice, M. Raineri and M Gallardo. Phys. Scr. T 134, 014018- 6pp (2009). doi: 10.1088/0031-8949/2009/T134/014018

[41] M. Raineri, M. Gallardo, S. Padilla and J. Reyna Almandos. J Phys B 42, 205004 6pp (2009). doi: 10.1088/0953-4075/42/20/205004

[42] M. Gallardo, M. Raineri, J. Reyna Almandos and É. Biémont. J Phys B 44, 045001- 7pp (2011). doi: 10.1088/0953-4075/44/4/045001

[43] M. Gallardo, M. Raineri, J. Reyna Almandos, C. J. B. Pagan and R. A. Abrahão. Ap.J.S.S. 216, 11, 2015. doi: 10.1088/0067-0049/216/1/11

[44] R.D. Cowan. The theory of atomic structure and spectra. University of California Press, Berkeley, CA. 1981.

[45] F. P. J Valero. J. Opt. Soc. Am. 58, 484 (1968). doi: 10.1364/JOSA.58.000484 


\title{
Canadian Journal of Physics
}

\author{
Page 8 of 21
}

https://mc06.manuscriptcentral.com/cjp-pubs 
Table 1 New and adjusted Xe VII and Xe VIII energy levels

\begin{tabular}{|c|c|c|c|c|c|c|c|}
\hline XeVII & Configuration & Term & $\mathbf{J}$ & $\begin{array}{c}\operatorname{Exp} \\
\text { Level } \\
\left(\mathrm{cm}^{-1}\right)\end{array}$ & $\begin{array}{c}\text { Fitted }^{(\mathrm{a})} \\
\left(\mathrm{cm}^{-1}\right)\end{array}$ & Composition $^{(\mathrm{b})}$ & Comment \\
\hline & $5 \mathrm{~s} 6 \mathrm{~d}$ & ${ }^{3} \mathrm{D}$ & 1 & 475997 & 475964 & 98 & Adjusted \\
\hline & & & 2 & 476250 & 476301 & 98 & Adjusted \\
\hline & & & 3 & 476785 & 476767 & 99 & Adjusted \\
\hline & & ${ }^{1} \mathrm{D}$ & 2 & 480945 & 480941 & 94 & New \\
\hline & $5 \mathrm{~s} 7 \mathrm{~s}$ & ${ }^{3} \mathrm{~S}$ & 1 & 506258 & 506258 & 99 & Adjusted \\
\hline & & ${ }^{\mathrm{T}} \mathrm{S}$ & 0 & 510325 & 510325 & 97 & New \\
\hline & $5 \mathrm{~s} 7 \mathrm{p}$ & ${ }^{1} \mathrm{P}^{\circ}$ & 1 & 531864 & 531536 & $865 s 7 p{ }^{1} \mathrm{P}+95 \mathrm{~s}^{2} \mathrm{p}^{3} \mathrm{P}$ & New \\
\hline & $4 d^{9} 5 s^{2} 5 p$ & ${ }^{3} \mathrm{P}^{\circ}$ & 1 & 529410 & 529502 & $734 d^{9} 5 s^{2} 5 p^{3} P+154 d^{9} 5 s^{2} 5 p^{3} D+85 s 7 p{ }^{3} P$ & New \\
\hline & & & 2 & 514393 & 514179 & $804 d^{9} 5 s^{2} 5 p^{3} P+154 d^{9} 5 s^{2} 5 p^{3} D$ & New \\
\hline & & ${ }^{3} \mathrm{D}^{\circ}$ & 2 & 552449 & 552378 & $584 d^{9} 5 s^{2} 5 p^{3} D+294 d^{9} 5 s^{2} 5 p{ }^{1} D+94 d^{9} 5 s^{2} 5 p^{3} F$ & New \\
\hline & & & 3 & 539708 & 539287 & $724 d^{9} 5 s^{2} 5 p^{3} D+284 d^{9} 5 s^{2} 5 p{ }^{1} F$ & New \\
\hline & & ${ }^{3} \mathrm{~F}^{\circ}$ & 3 & 517693 & 517747 & $554 d^{9} 5 s^{2} 5 p^{3} F+334 d^{9} 5 s^{2} 5 p{ }^{1} F+124 d^{9} 5 s^{2} 5 p^{3} D$ & New \\
\hline & & & 4 & 530874 & 531027 & 100 & New \\
\hline & & ${ }^{1} \mathrm{D}^{\circ}$ & 2 & 536687 & 537132 & $664 d^{9} 5 s^{2} 5 p{ }^{1} D+204 d^{9} 5 s^{2} 5 p^{3} D+124 d^{9} 5 s^{2} 5 p^{3} P$ & New \\
\hline
\end{tabular}




\begin{tabular}{|c|c|c|c|c|c|c|c|}
\hline Xe VIII & $4 d^{9} 5 \mathrm{~s} 5 \mathrm{~d} \quad\left({ }^{3} \mathrm{D}\right)$ & ${ }^{4} \mathrm{P}$ & $5 / 2$ & 749456 & 749568 & $654 d^{9} 5 s 5 d^{4} P+324 d^{9} 5 s 5 d^{4} D$ & New \\
\hline & $\left({ }^{3} \mathrm{D}\right)$ & ${ }^{4} \mathrm{~F}$ & $5 / 2$ & 755624 & 755510 & $294 d^{9} 5 s 5 d^{4} F+184 d^{9} 5 s 5 d^{4} D+164 d^{9} 5 s 5 d^{4} G$ & New \\
\hline & $\left({ }^{3} \mathrm{D}\right)$ & ${ }^{4} \mathrm{P}$ & $1 / 2$ & 757388 & 757364 & $454 d^{9} 5 s 5 d^{4} P+194 d^{9} 5 s 5 d\left({ }^{1} D\right){ }^{2} P+144 d^{9} 5 s 5 d^{2} P$ & New \\
\hline & $\left({ }^{3} \mathrm{D}\right)$ & ${ }^{4} \mathrm{D}$ & $1 / 2$ & 767993 & 767718 & $584 d^{9} 5 s 5 d^{4} D+174 d^{9} 5 s 5 d^{2} P+124 d^{9} 5 s 5 d\left({ }^{1} D\right){ }^{2} P$ & New \\
\hline & $\left({ }^{3} \mathrm{D}\right)$ & ${ }^{4} \mathrm{D}$ & $3 / 2$ & 769280 & 769547 & $474 d^{9} 5 s 5 d^{4} D+164 d^{9} 5 s 5 d^{4} F+104 d^{9} 5 s 5 d\left({ }^{1} D\right){ }^{2} D$ & New \\
\hline & $\left({ }^{1} \mathrm{D}\right)$ & ${ }^{2} \mathrm{~S}$ & $1 / 2$ & 769667 & 769705 & $484 d^{9} 5 s 5 d\left({ }^{1} D\right){ }^{2} S+174 d^{9} 5 s 5 d\left({ }^{1} D\right){ }^{2} P+104 d^{9} 5 s 5 d{ }^{2} S$ & New \\
\hline & $\left({ }^{3} \mathrm{D}\right)$ & ${ }^{2} \mathrm{P}$ & $1 / 2$ & 788973 & 788972 & $474 d^{9} 5 s 5 d{ }^{2} P+254 d^{9} 5 s 5 d\left({ }^{1} D\right){ }^{2} P+134 d^{9} 5 s 5 d\left({ }^{1} D\right){ }^{2} S$ & New \\
\hline
\end{tabular}

${ }^{a}$ Calculated energy level values obtained using the fitted energy parameters

${ }^{\mathrm{b}}$ Percentages below $5 \%$ have been omitted 
Table2 New classified lines of Xe VII and Xe VIII

\begin{tabular}{|c|c|c|c|c|c|c|c|c|c|c|c|}
\hline \multirow{3}{*}{$\begin{array}{l}\text { Ion } \\
\\
\text { VII }\end{array}$} & \multirow{3}{*}{\begin{tabular}{|r|} 
Rel. \\
Int. \\
1 \\
\end{tabular}} & \multirow{3}{*}{$\begin{array}{c}\begin{array}{c}\text { Observed } \\
\text { Wavelength } \\
\text { Vac }(\AA)\end{array} \\
427.96\end{array}$} & \multicolumn{2}{|c|}{$\begin{array}{c}\text { Wavenumber } \\
\text { (cm-1) }\end{array}$} & \multirow{2}{*}{\multicolumn{3}{|c|}{$\begin{array}{l}\text { Lower } \quad \text { Level } \\
\text { Conf. Term, J }\end{array}$}} & \multirow{2}{*}{\multicolumn{3}{|c|}{$\begin{array}{l}\text { Upper } \quad \text { Level } \\
\text { Conf. Term, J }\end{array}$}} & \multirow{3}{*}{\begin{tabular}{|c|}
$\mathbf{g A}\left(\mathbf{s}^{-1}\right)$ \\
$3.58 \times 10^{6}$
\end{tabular}} \\
\hline & & & $\sigma_{\text {obs }}$ & $\sigma_{\text {cal }}$ & & & & & & & \\
\hline & & & 233667 & 233677 & $4 \mathrm{f} 5 \mathrm{~s}$ & ${ }^{3} \mathrm{~F}$ & 2 & $5 \mathrm{~s} 7 \mathrm{~s}$ & ${ }^{3} \mathrm{~S}$ & 1 & \\
\hline VII & 1 & 445.81 & 224311 & 224322 & $5 \mathrm{~s} 5 \mathrm{~d}$ & ${ }^{1} \mathrm{D}$ & 2 & $5 s 7 p$ & ${ }^{1} \mathrm{P}$ & 1 & $5.60 \times 10^{9}$ \\
\hline VII & 6 & 489.74 & 204190 & 204204 & $4 f 5 s$ & ${ }^{3} \mathrm{~F}$ & 2 & $5 s 6 d$ & ${ }^{3} \mathrm{D}$ & 3 & $1.04 \times 10^{6}$ \\
\hline VII & 3 & 490.96 & 203683 & 203669 & $4 \mathrm{f} 5 \mathrm{~s}$ & $\sqrt[3]{F}$ & 2 & $5 \mathrm{~s} 6 \mathrm{~d}$ & ${ }^{3} \mathrm{D}$ & 2 & $2.77 \times 10^{7}$ \\
\hline VII & 4 & 587.54 & 217001 & 216193 & $5 \mathrm{~s} 6 \mathrm{~s}$ & ${ }^{1} \mathrm{~S}$ & 0 & $5 s 7 p$ & ${ }^{1} \mathrm{P}$ & 1 & $5.62 \times 10^{7}$ \\
\hline VII & 1 & 603.74 & 165634 & 165638 & $4 f 5 p$ & ${ }^{3} \mathrm{~F}$ & 2 & $4 d^{9} 5 s^{2} 5 p$ & ${ }^{3} \mathrm{D}$ & 2 & $6.65 \times 10^{5}$ \\
\hline VII & 4 & 647.61 & 154414 & 154422 & $4 f 5 p$ & ${ }^{1} \mathrm{~F}$ & 3 & $4 d^{9} 5 s^{2} 5 p$ & ${ }^{3} \mathrm{D}$ & 2 & $1.26 \times 10^{3}$ \\
\hline VII & 3 & 654.02 & 152901 & 152897 & $4 f 5 p$ & ${ }^{3} \mathrm{~F}$ & 2 & $4 d^{9} 5 s^{2} 5 p$ & $\mathrm{D}$ & 3 & $3.24 \times 10^{4}$ \\
\hline VII & 3 & 661.09 & 151265 & 151265 & $4 f 5 p$ & ${ }^{3} \mathrm{~F}$ & 3 & $4 d^{9} 5 s^{2} 5 p$ & ${ }^{1} \mathrm{D}$ & 2 & $4.41 \times 10^{5}$ \\
\hline VII & 4 & 673.35 & 148511 & 148518 & $4 f 5 p$ & ${ }^{3} \mathrm{G}$ & 3 & $4 d^{9} 5 s^{2} 5 p$ & $\mathrm{~F}$ & 4 & $3.48 \times 10^{4}$ \\
\hline VII & 5 & 687.53 & 145448 & 145452 & $4 f 5 p$ & ${ }^{3} \mathrm{~F}$ & 3 & $4 d^{9} 5 s^{2} 5 p$ & ${ }^{3} \mathrm{~F}$ & 4 & $4.42 \times 10^{6}$ \\
\hline VII & 4 & 715.70 & 139723 & 139721 & $4 f 5 p$ & ${ }^{3} \mathrm{~F}$ & 4 & $4 d^{9} 5 s^{2} 5 p$ & ${ }^{3} \mathrm{D}$ & 3 & $1.21 \times 10^{6}$ \\
\hline VII & 5 & 721.19 & 138660 & 138660 & $4 f 5 p$ & ${ }^{\mathrm{T}} \mathrm{F}$ & 3 & $4 d^{9} 5 s^{2} 5 p$ & ${ }^{\mathrm{I}} \mathrm{D}$ & 2 & $1.51 \times 10^{6}$ \\
\hline
\end{tabular}




\begin{tabular}{|c|c|c|c|c|c|c|c|c|c|c|c|}
\hline VII & 5 & 734.83 & 136086 & 136092 & $4 f 5 p$ & ${ }^{1} \mathrm{D}$ & 2 & $4 d^{9} 5 s^{2} 5 p$ & ${ }^{3} \mathrm{D}$ & 2 & $3.22 \times 10^{7}$ \\
\hline VII & 2 & 742.23 & 134729 & 134729 & $4 f 5 p$ & ${ }^{3} \mathrm{D}$ & 3 & $4 d^{9} 5 s^{2} 5 p$ & ${ }^{3} \mathrm{D}$ & 3 & $1.65 \times 10^{6}$ \\
\hline VII & 5 & 756.03 & 132270 & 132271 & $4 f 5 p$ & ${ }^{3} \mathrm{~F}$ & 3 & $4 d^{9} 5 s^{2} 5 p$ & ${ }^{3} \mathrm{~F}$ & 3 & $3.66 \times 10^{6}$ \\
\hline VII & 7 & 764.02 & 130887 & 130882 & $4 f 5 p$ & ${ }^{3} \mathrm{~F}$ & 2 & $4 d^{9} 5 s^{2} 5 p$ & ${ }^{3} \mathrm{~F}$ & 3 & $2.61 \times 10^{7}$ \\
\hline VII & 7 & 764.02 & 130887 & 130887 & $4 f 5 p$ & ${ }^{3} \mathrm{~F}$ & 4 & $4 d^{9} 5 s^{2} 5 p$ & ${ }^{3} \mathrm{~F}$ & 4 & $2.10 \times 10^{7}$ \\
\hline VII & 1 & 775.37 & 128971 & 128971 & $4 f 5 p$ & ${ }^{3} \mathrm{~F}$ & 3 & $4 d^{9} 5 s^{2} 5 p$ & ${ }^{3} \mathrm{P}$ & 2 & $5.49 \times 10^{6}$ \\
\hline VII & 5 & 781.76 & 127917 & 127920 & $4 f 5 p$ & ${ }^{3} \mathrm{D}$ & 1 & $4 d^{9} 5 s^{2} 5 p$ & ${ }^{1} \mathrm{D}$ & 2 & $2.69 \times 10^{4}$ \\
\hline VII & 2 & 812.56 & 123068 & 123068 & $4 f 5 p$ & ${ }^{3} \mathrm{D}$ & 2 & $4 d^{9} 5 s^{2} 5 p$ & ${ }^{3} \mathrm{P}$ & 1 & $3.29 \times 10^{6}$ \\
\hline VII & 5 & 828.91 & 120640 & 120643 & $4 f 5 p$ & ${ }^{3} \mathrm{D}$ & 1 & $4 d^{9} 5 s^{2} 5 p$ & ${ }^{3} \mathrm{P}$ & 1 & $1.91 \times 10^{6}$ \\
\hline VII & 3 & 831.03 & 120333 & 120330 & $4 f 5 p$ & ${ }^{1} \mathrm{D}$ & 2 & $4 d^{9} 5 s^{2} 5 p$ & ${ }^{1} \mathrm{D}$ & 2 & $1.44 \times 10^{5}$ \\
\hline VII & 5 & 859.33 & 116370 & 116366 & $4 f 5 p$ & ${ }^{1} \mathrm{~F}$ & 3 & $4 d^{9} 5 s^{2} 5 p$ & ${ }^{3} \mathrm{P}$ & 2 & $2.07 \times 10^{5}$ \\
\hline VII & 4 & 884.51 & 113057 & 113053 & $4 f 5 p$ & ${ }^{1} \mathrm{D}$ & 2 & $4 d^{9} 5 s^{2} 5 p$ & ${ }^{3} \mathrm{P}$ & 1 & $2.17 \times 10^{6}$ \\
\hline VII & 6 & 887.20 & 112714 & 112714 & $4 f 5 p$ & ${ }^{3} \mathrm{D}$ & 3 & $4 d^{9} 5 s^{2} 5 p$ & ${ }^{3} \mathrm{~F}$ & 3 & $1.69 \times 10^{6}$ \\
\hline VII & 8 & 898.03 & 111355 & 111351 & $4 f 5 p$ & ${ }^{3} \mathrm{D}$ & 2 & $4 d^{9} 5 s^{2} 5 p$ & ${ }^{3} \mathrm{~F}$ & 3 & $6.41 \times 10^{5}$ \\
\hline VII & 7 & 925.51 & 108049 & 108051 & $4 f 5 p$ & ${ }^{3} \mathrm{D}$ & 2 & $4 d^{9} 5 s^{2} 5 p$ & ${ }^{3} \mathrm{P}$ & 2 & $8.63 \times 10^{5}$ \\
\hline VII & 7 & 942.16 & 106139 & 106142 & $4 f 5 p$ & ${ }^{1} \mathrm{G}$ & 4 & $4 d^{9} 5 s^{2} 5 p$ & ${ }^{3} \mathrm{~F}$ & 3 & $3.76 \times 10^{8}$ \\
\hline VII & 1 & 947.05 & 105591 & 105592 & $5 s 6 p$ & ${ }^{3} \mathrm{P}$ & 0 & $5 \mathrm{~s} 7 \mathrm{~s}$ & ${ }^{3} \mathrm{~S}$ & 1 & $3.20 \times 10^{9}$ \\
\hline VII & 4 & 975.38 & 102524 & 102523 & $5 \mathrm{~s} 6 \mathrm{p}$ & ${ }^{1} \mathrm{P}$ & 1 & $5 \mathrm{~s} 7 \mathrm{~s}$ & ${ }^{1} \mathrm{~S}$ & 0 & $2.73 \times 10^{9}$ \\
\hline
\end{tabular}




\begin{tabular}{|c|c|c|c|c|c|c|c|c|c|c|c|}
\hline VII & 4 & 1212.73 & 82459 & 82458 & $5 \mathrm{p} 5 \mathrm{~d}$ & ${ }^{3} \mathrm{~F}$ & 2 & $5 \mathrm{~s} 6 \mathrm{~d}$ & ${ }^{3} \mathrm{D}$ & 2 & $1.36 \times 10^{8}$ \\
\hline VII & 7 & 1234.17 & 81026 & 81024 & $5 \mathrm{p} 5 \mathrm{~d}$ & ${ }^{3} \mathrm{P}$ & 2 & $5 \mathrm{~s} 7 \mathrm{~s}$ & ${ }^{3} \mathrm{~S}$ & 1 & $1.84 \times 10^{8}$ \\
\hline VII & 1 & 1321.64 & 75664 & 75663 & $5 \mathrm{~s} 6 \mathrm{~d}$ & ${ }^{3} \mathrm{D}$ & 3 & $4 d^{9} 5 s^{2} 5 p$ & ${ }^{3} \mathrm{D}$ & 2 & $4.28 \times 10^{5}$ \\
\hline VII & 2 & 1460.95 & 68449 & 68448 & $5 s 6 p$ & ${ }^{1} \mathrm{P}$ & 1 & $5 \mathrm{~s} 6 \mathrm{~d}$ & ${ }^{3} \mathrm{D}$ & 2 & $1.18 \times 10^{9}$ \\
\hline VII & 2 & 1539.03 & 64976 & 64975 & $5 \mathrm{p} 5 \mathrm{~d}$ & ${ }^{3} \mathrm{D}$ & 1 & $5 \mathrm{~s} 6 \mathrm{~d}$ & ${ }^{3} \mathrm{D}$ & 1 & $1.16 \times 10^{8}$ \\
\hline VII & 1 & 1541.24 & 64883 & 64882 & $5 \mathrm{p} 5 \mathrm{~d}$ & ${ }^{1} \mathrm{P}$ & 1 & $5 \mathrm{~s} 7 \mathrm{~s}$ & ${ }^{3} \mathrm{~S}$ & 1 & $3.96 \times 10^{6}$ \\
\hline VII & 7 & 1557.13 & 64221 & 64218 & $5 \mathrm{p} 5 \mathrm{~d}$ & ${ }^{3} \mathrm{~F}$ & 4 & $5 \mathrm{~s} 6 \mathrm{~d}$ & ${ }^{3} \mathrm{D}$ & 3 & $9.51 \times 10^{8}$ \\
\hline VII & 7 & 1726.57 & 57918 & 57917 & $5 \mathrm{p} 5 \mathrm{~d}$ & ${ }^{3} \mathrm{D}$ & 3 & $5 \mathrm{~s} 6 \mathrm{~d}$ & ${ }^{1} \mathrm{D}$ & 2 & $1.27 \times 10^{8}$ \\
\hline VII & 7 & 1963.86 & 50920 & 50919 & $5 \mathrm{~s} 6 \mathrm{~d}$ & ${ }^{1} \mathrm{D}$ & 2 & $5 s 7 p$ & ${ }^{1} \mathrm{P}$ & 1 & $3.44 \times 10^{9}$ \\
\hline VII & 9 & 4641.31 & 21540 & 21539 & $5 \mathrm{~s} 7 \mathrm{~s}$ & ${ }^{1} \mathrm{~S}$ & 0 & $5 s 7 p$ & ${ }^{1} \mathrm{P}$ & 1 & $2.45 \times 10^{8}$ \\
\hline & & $\begin{array}{c}\text { Observed } \\
\text { Wavelength } \\
\text { Vac }(\AA)\end{array}$ & & & & & & & & & \\
\hline VIII & 8 & 419.22 & 238538 & 238524 & $4 d^{9} 5 s 5 p$ & ${ }^{4} \mathrm{~F}$ & $3 / 2$ & $4 d^{9} 5 s 5 d$ & $\left({ }^{3} \mathrm{D}\right)^{2} \mathrm{P}$ & $1 / 2$ & $4.55 \times 10^{8}$ \\
\hline VIII & 2 & 430.34 & 232374 & 232364 & $4 d^{9} 5 s 5 p$ & $\left({ }^{1} \mathrm{D}\right)^{2} \mathrm{D}$ & $3 / 2$ & $4 d^{9} 5 \mathrm{~s} 5 \mathrm{~d}$ & $\left({ }^{3} \mathrm{D}\right)^{2} \mathrm{P}$ & $1 / 2$ & $1.05 \times 10^{10}$ \\
\hline VIII & 1 & 439.13 & 227723 & 227725 & $4 d^{9} 5 s 5 p$ & ${ }^{4} \mathrm{P}$ & $3 / 2$ & $4 d^{9} 5 \mathrm{~s} 5 d$ & ${ }^{4} \mathrm{D}$ & $3 / 2$ & $2.67 \times 10^{10}$ \\
\hline VIII & 1 & 441.62 & 226439 & 226438 & $4 d^{9} 5 s 5 p$ & ${ }^{4} \mathrm{P}$ & $3 / 2$ & $4 d^{9} 5 \mathrm{~s} 5 d$ & ${ }^{4} \mathrm{D}$ & $1 / 2$ & $2.24 \times 10^{9}$ \\
\hline VIII & 4 & 444.78 & 224830 & 224824 & $4 d^{9} 5 s^{9} p$ & $\left({ }^{1} \mathrm{D}\right)^{2} \mathrm{P}$ & $1 / 2$ & $4 d^{9} 5 s^{5} d$ & $\left({ }^{3} \mathrm{D}\right)^{2} \mathrm{P}$ & $1 / 2$ & $3.96 \times 10^{8}$ \\
\hline
\end{tabular}




\begin{tabular}{|c|c|c|c|c|c|c|c|c|c|c|c|}
\hline VIII & 4 & 459.70 & 217533 & 217544 & $4 d^{9} 5 s 5 p$ & ${ }^{4} \mathrm{~F}$ & $3 / 2$ & $4 d^{9} 5 s 5 d$ & ${ }^{4} \mathrm{D}$ & $1 / 2$ & $2.70 \times 10^{9}$ \\
\hline VIII & 1 & 463.32 & 215834 & 215833 & $4 d^{9} 5 s 5 p$ & ${ }^{4} \mathrm{P}$ & $3 / 2$ & $4 d^{9} 5 s 5 d$ & ${ }^{4} \mathrm{P}$ & $1 / 2$ & $3.80 \times 10^{10}$ \\
\hline VIII & 2 & 470.23 & 212662 & 212671 & $4 d^{9} 5 s 5 p$ & $\left({ }^{1} D\right)^{2} D$ & $3 / 2$ & $4 d^{9} 5 s 5 d$ & ${ }^{4} \mathrm{D}$ & $3 / 2$ & $1.30 \times 10^{9}$ \\
\hline VIII & 4 & 473.05 & 211394 & 211384 & $4 d^{9} 5 \mathrm{~s} 5 p$ & $\left({ }^{1} \mathrm{D}\right)^{2} \mathrm{D}$ & $3 / 2$ & $4 d^{9} 5 s 5 d$ & ${ }^{4} \mathrm{D}$ & $1 / 2$ & $1.42 \times 10^{9}$ \\
\hline VIII & $2 \mathrm{bl}$ & 480.98 & 207909 & 207901 & $4 d^{9} 5 s 5 p$ & ${ }^{4} \mathrm{P}$ & $3 / 2$ & $4 d^{9} 5 \mathrm{~s} 5 \mathrm{~d}$ & ${ }^{4} \mathrm{P}$ & $5 / 2$ & $1.32 \times 10^{9}$ \\
\hline VIII & 3 & 483.22 & 206945 & 206939 & $4 d^{9} 5 s 5 p$ & ${ }^{4} \mathrm{~F}$ & $3 / 2$ & $4 d^{9} 5 \mathrm{~s} 5 \mathrm{~d}$ & ${ }^{4} \mathrm{P}$ & $1 / 2$ & $1.27 \times 10^{9}$ \\
\hline VIII & 3 & 483.22 & 206945 & 206937 & $4 d^{9} 5 s 5 p$ & $\left({ }^{1} \mathrm{D}\right)^{2} \mathrm{P}$ & $3 / 2$ & $4 d^{9} 5 \mathrm{~s} 5 \mathrm{~d}$ & $\left({ }^{1} D\right)^{2} S$ & $1 / 2$ & $7.27 \times 10^{9}$ \\
\hline VIII & 4 & 487.40 & 205170 & 205175 & $4 d^{9} 5 s 5 p$ & ${ }^{4} \mathrm{~F}$ & $3 / 2$ & $4 d^{9} 5 s 5 d$ & ${ }^{4} \mathrm{~F}$ & $5 / 2$ & $2.00 \times 10^{7}$ \\
\hline VIII & 3 & 490.96 & 203683 & 203685 & $4 d^{9} 5 s 5 p$ & $\left({ }^{3} \mathrm{D}\right)^{2} \mathrm{P}$ & $3 / 2$ & $4 d^{9} 5 s 5 d$ & $\left({ }^{3} \mathrm{D}\right)^{2} \mathrm{P}$ & $1 / 2$ & $1.07 \times 10^{10}$ \\
\hline VIII & 1 & 497.31 & 201082 & 201072 & $4 d^{9} 5 s 5 p$ & חי & $1 / 2$ & $4 d^{9} 5 s 5 d$ & $\left({ }^{1} \mathrm{D}\right)^{2} \mathrm{~S}$ & $1 / 2$ & $3.05 \times 10^{7}$ \\
\hline VIII & 4 & 498.04 & 200787 & 200779 & $4 d^{9} 5 \mathrm{~s} 5 \mathrm{p}$ & $\left({ }^{1} D\right)^{2} D$ & $3 / 2$ & $4 d^{9} 5 s 5 d$ & ${ }^{4} \mathrm{P}$ & $1 / 2$ & $1.06 \times 10^{8}$ \\
\hline VIII & 2 & 501.51 & 199398 & 199398 & $4 d^{9} 5 s 5 p$ & ${ }^{4} \mathrm{D}$ & $1 / 2$ & $4 d^{9} 5 s 5 d$ & ${ }^{4} \mathrm{D}$ & $1 / 2$ & $1.86 \times 10^{9}$ \\
\hline VIII & 7 & 502.50 & 199005 & 199007 & $4 d^{9} 5 s 5 p$ & ${ }^{4} \mathrm{~F}$ & $3 / 2$ & $4 d^{9} 5 \mathrm{~s} 5 \mathrm{~d}$ & ${ }^{4} \mathrm{P}$ & $5 / 2$ & $2.78 \times 10^{8}$ \\
\hline VIII & 7 & 502.50 & 199005 & 199015 & $4 d^{9} 5 s 5 p$ & $\left({ }^{1} \mathrm{D}\right)^{2} \mathrm{D}$ & $3 / 2$ & $4 d^{9} 5 \mathrm{~s} 5 d$ & ${ }^{4} \mathrm{~F}$ & $5 / 2$ & $2.00 \times 10^{10}$ \\
\hline VIII & 2 & 512.68 & 195053 & 195052 & $4 d^{9} 5 s 5 p$ & ${ }^{4} \mathrm{D}$ & $3 / 2$ & $4 d^{9} 5 s 5 d$ & ${ }^{4} \mathrm{D}$ & $3 / 2$ & $3.41 \times 10^{8}$ \\
\hline VIII & 4 & 513.69 & 194670 & 194658 & $4 d^{9} 5 \mathrm{~s} 5 \mathrm{p}$ & $\left({ }^{1} D\right)^{2} P$ & $3 / 2$ & $4 d^{9} 5 s 5 d$ & ${ }^{4} \mathrm{P}$ & $1 / 2$ & $4.21 \times 10^{7}$ \\
\hline VIII & 1 & 517.49 & 193241 & 193239 & $4 d^{9} 5 s 5 p$ & $\left({ }^{1} \mathrm{D}\right)^{2} \mathrm{P}$ & $1 / 2$ & $4 d^{9} 5 s 5 d$ & ${ }^{4} \mathrm{P}$ & $1 / 2$ & $2.10 \times 10^{9}$ \\
\hline VIII & 2 & 542.36 & 184379 & 184379 & $4 d^{9} 5 \mathrm{~s} 5 p$ & $\left({ }^{3} \mathrm{D}\right)^{2} \mathrm{P}$ & $3 / 2$ & $4 d^{9} 5 s 5 d$ & $\left({ }^{1} \mathrm{D}\right)^{2} \mathrm{~S}$ & $1 / 2$ & $2.14 \times 10^{10}$ \\
\hline
\end{tabular}




\begin{tabular}{|c|c|c|c|c|c|c|c|c|c|c|c|}
\hline VIII & 6 & 547.35 & 182698 & 182705 & $4 d^{9} 5 \mathrm{~s} 5 \mathrm{p}$ & $\left({ }^{3} \mathrm{D}\right)^{2} \mathrm{P}$ & $3 / 2$ & $4 \mathrm{~d}^{9} 5 \mathrm{~s} 5 \mathrm{~d}$ & ${ }^{4} \mathrm{D}$ & $1 / 2$ & $4.26 \times 10^{9}$ \\
\hline VIII & 2 & 551.27 & 181399 & 181396 & $4 \mathrm{~d}^{9} 5 \mathrm{~s} 5 \mathrm{p}$ & ${ }^{4} \mathrm{D}$ & $3 / 2$ & $4 \mathrm{~d}^{9} 5 \mathrm{~s} 5 \mathrm{~d}$ & ${ }^{4} \mathrm{~F}$ & $5 / 2$ & $1.33 \times 10^{10}$ \\
\hline VIII & 6 & 1614.37 & 61944 & 61944 & $4 \mathrm{~d}^{9} 5 \mathrm{~s} 5 \mathrm{~d}$ & ${ }^{4} \mathrm{P}$ & $5 / 2$ & $4 \mathrm{~d}^{9} 5 \mathrm{~s} 4 \mathrm{f}$ & ${ }^{2} \mathrm{P}$ & $3 / 2$ & $5.22 \times 10^{8}$ \\
\hline
\end{tabular}

Note: bl, blended line 
Table 3 Energy parameters $\left(\mathrm{cm}^{-1}\right)$ for the studied even parity configurations of Xe VII

\begin{tabular}{|c|c|c|c|c|}
\hline Configuration & Parameter & H-F value & Fitted value & Fitt/H-F \\
\hline $5 s^{2}$ & $\mathrm{E}_{\mathrm{av}}$ & 0 & $7009 \pm 174$ & \\
\hline \multirow[t]{4}{*}{$5 p^{2}$} & $\mathrm{E}_{\mathrm{av}}$ & 238023 & $248510 \pm 143$ & 1.04 \\
\hline & $F^{2}(5 p, 5 p)$ & 59078 & $53119 \pm 1789$ & 0.90 \\
\hline & $\alpha$ & 0 & 545 (FIX) & \\
\hline & $\zeta_{5 \mathrm{p}}$ & 10548 & $11632 \pm 93$ & 1.10 \\
\hline \multirow[t]{3}{*}{$5 \mathrm{~s} 5 \mathrm{~d}$} & $\mathrm{E}_{\mathrm{av}}$ & 283684 & $293214 \pm 97$ & 1.03 \\
\hline & $\zeta_{5 \mathrm{~d}}$ & 842 & $1064 \pm 95$ & 1.26 \\
\hline & $G^{2}(5 s, 5 d)$ & 38699 & $26141 \pm 655$ & 0.68 \\
\hline \multirow[t]{3}{*}{$5 \mathrm{~s} 6 \mathrm{~d}$} & $\mathrm{E}_{\mathrm{av}}$ & 467624 & $478435 \pm 90$ & 0.98 \\
\hline & $\zeta_{6 \mathrm{~d}}$ & 377 & 358 & 0.95 \\
\hline & $\mathrm{G}^{2}(5 \mathrm{~s}, 6 \mathrm{~d})$ & 9108 & $11945 \pm 527$ & 1.31 \\
\hline $5 \mathrm{~s} 7 \mathrm{~d}$ & $\mathrm{E}_{\mathrm{av}}$ & 555672 & 555672 (FIX) & 1.00 \\
\hline $5 \mathrm{~s} 8 \mathrm{~d}$ & $\mathrm{E}_{\mathrm{av}}$ & 605411 & 605411 (FIX) & 1.00 \\
\hline \multirow[t]{2}{*}{$5 \mathrm{~s} 6 \mathrm{~s}$} & $\mathrm{E}_{\mathrm{av}}$ & 347141 & $358325 \pm 135$ & 1.03 \\
\hline & $G^{0}(5 s, 6 s)$ & 5194 & $4160 \pm 121$ & 0.80 \\
\hline \multirow[t]{2}{*}{$5 \mathrm{~s} 7 \mathrm{~s}$} & $\mathrm{E}_{\mathrm{av}}$ & 494169 & $507618 \pm 135$ & 1.03 \\
\hline & $\mathrm{G}^{0}(5 \mathrm{~s}, 7 \mathrm{~s})$ & 1789 & $2464 \pm 122$ & 1.40 \\
\hline \multirow[t]{6}{*}{$4 f 5 p$} & $\mathrm{E}_{\mathrm{av}}$ & 388140 & $398510 \pm 50$ & 1.03 \\
\hline & $\zeta_{4 \mathrm{f}}$ & 254 & $202 \pm 36$ & 0.80 \\
\hline & $\zeta_{5 \mathrm{p}}$ & 10135 & $11505 \pm 97$ & 1.14 \\
\hline & $F^{2}(4 f, 5 p)$ & 51181 & $42077 \pm 621$ & 0.82 \\
\hline & $G^{2}(4 f, 5 p)$ & 35098 & $29717 \pm 441$ & 0.85 \\
\hline & $G^{4}(4 f, 5 p)$ & 26034 & $19416 \pm 635$ & 0.75 \\
\hline $5 f 5 p$ & $\mathrm{E}_{\mathrm{av}}$ & 577384 & 577384 (FIX) & 1.00 \\
\hline $5 p 6 p$ & $\mathrm{E}_{\mathrm{av}}$ & 521670 & 521670 (FIX) & 1.00 \\
\hline $4 f^{2}$ & $\mathrm{E}_{\mathrm{av}}$ & 546646 & 546646 (FIX) & 1.00 \\
\hline $5 d^{2}$ & $\mathrm{E}_{\mathrm{av}}$ & 581986 & 581986 (FIX) & 1.00 \\
\hline $5 s^{2} 5 d$ & $\mathrm{E}_{\mathrm{av}}$ & 721545 & 721545 (FIX) & 1.00 \\
\hline
\end{tabular}




$$
5 \mathrm{~s}^{2} 6 \mathrm{~s} \quad \mathrm{E}_{\mathrm{av}} \quad 788768 \quad 788768 \text { (FIX) } \quad 1.00
$$

${ }^{a}$ Parameters omitted from this table: direct and exchange integrals, and spin-orbit $\zeta$ parameters set to $85 \%$ and $95 \%$ of their HFR values respectively; CI integrals were set to $85 \%$ of their HFR values. The standard deviation for the energy adjustment was 175 $\mathrm{cm}^{-1}$.

Table 4 Energy parameters $\left(\mathrm{cm}^{-1}\right)$ for the studied odd parity configurations of Xe VII

\begin{tabular}{|c|c|c|c|c|}
\hline Configuration & Parameter & $\mathrm{H}$-F value & Fitted value & Fitt/H-F \\
\hline \multirow[t]{3}{*}{$5 \mathrm{~s} 5 \mathrm{p}$} & $\mathrm{E}_{\mathrm{av}}$ & 108319 & $118939 \pm 154$ & 1.10 \\
\hline & $\zeta_{5 \mathrm{p}}$ & 10622 & $11694 \pm 246$ & 1.10 \\
\hline & $G^{1}(5 s, 5 p)$ & 76747 & $62102 \pm 579$ & 0.81 \\
\hline \multirow[t]{3}{*}{$5 s 6 p$} & $\mathrm{E}_{\mathrm{av}}$ & 396097 & $407212 \pm 92$ & 1.03 \\
\hline & $\zeta_{6 \mathrm{p}}$ & 3931 & $4626 \pm 260$ & 1.17 \\
\hline & $G^{1}(5 s, 6 p)$ & 8539 & $6315 \pm 903$ & 0.74 \\
\hline \multirow[t]{3}{*}{$5 \mathrm{~s} 7 \mathrm{p}$} & $\mathrm{E}_{\mathrm{av}}$ & 518483 & $529156 \pm 161$ & 1.02 \\
\hline & $\zeta_{7 \mathrm{p}}$ & 1975 & $1593 \pm 221$ & 0.81 \\
\hline & $G^{1}(5 s, 7 p)$ & 3176 & 2699(FIX) & 0.85 \\
\hline \multirow[t]{3}{*}{$5 \mathrm{~s} 4 \mathrm{f}$} & $\mathrm{E}_{\mathrm{av}}$ & 268592 & $275519 \pm 144$ & 1.03 \\
\hline & $\zeta_{4 \mathrm{f}}$ & 245 & 233 (FIX) & 0.95 \\
\hline & $G^{3}(5 s, 4 f)$ & 36153 & $29814 \pm 1172$ & 0.82 \\
\hline \multirow[t]{3}{*}{$6 s 4 f$} & $\mathrm{E}_{\mathrm{av}}$ & 615059 & 615059 (FIX) & 1.00 \\
\hline & $\zeta_{4 \mathrm{f}}$ & 271 & 258 (FIX) & 0.95 \\
\hline & $G^{3}(6 s, 4 f)$ & 6425 & 5461 (FIX) & 0.85 \\
\hline \multirow[t]{3}{*}{$5 \mathrm{~s} 5 \mathrm{f}$} & $\mathrm{E}_{\mathrm{av}}$ & 452423 & $461307 \pm 154$ & 1.02 \\
\hline & $\zeta_{5 f}$ & 87 & 87 (FIX) & 1.00 \\
\hline & $G^{3}(5 s, 5 f)$ & 4396 & $4854 \pm 1391$ & 1.10 \\
\hline \multirow[t]{5}{*}{$5 \mathrm{p} 5 \mathrm{~d}$} & $\mathrm{E}_{\mathrm{av}}$ & 406352 & $418416 \pm 95$ & 1.03 \\
\hline & $\zeta_{5 \mathrm{p}}$ & 10741 & $11387 \pm 239$ & 1.06 \\
\hline & $\zeta_{5 \mathrm{~d}}$ & 861 & $1495 \pm 151$ & 1.73 \\
\hline & $\mathrm{F}^{2}(5 \mathrm{p}, 5 \mathrm{~d})$ & 49703 & $39181 \pm 1042$ & 0.79 \\
\hline & $\mathrm{G}^{1}(5 \mathrm{p}, 5 \mathrm{~d})$ & 58495 & $43670 \pm 503$ & 0.73 \\
\hline
\end{tabular}




$\begin{array}{rllll} & \mathrm{G}^{3}(5 \mathrm{p}, 5 \mathrm{~d}) & 37389 & 27187 \pm 1042 & 0.73 \\ 5 \mathrm{p} 6 \mathrm{~s} & \mathrm{E}_{\mathrm{av}} & 473884 & 480754 \pm 154 & 1.01 \\ & \zeta_{5 \mathrm{p}} & 11075 & 11175 \pm 221 & 1.01 \\ & \mathrm{G}^{1}(5 \mathrm{p}, 6 \mathrm{~s}) & 7801 & 7627 \pm 750 & 0.98 \\ 4 \mathrm{f5d} & \mathrm{E}_{\mathrm{av}} & 552712 & 552712(\mathrm{FIX}) & 1.00 \\ 4 \mathrm{~d}^{9} 5 \mathrm{~s}^{2} 5 \mathrm{p} & \mathrm{E}_{\mathrm{av}} & 532109 & 534424 \pm 105 & 1.01 \\ & \zeta_{4 d} & 6274 & 5497 \pm 119 & 0.88 \\ & \zeta_{5 \mathrm{p}} & 11481 & 10924 \pm 230 & 0.95 \\ & \mathrm{~F}^{2}(4 \mathrm{~d}, 5 \mathrm{p}) & 41900 & 37782 \pm 1146 & 0.90 \\ & \mathrm{G}^{1}(4 \mathrm{~d}, 5 \mathrm{p}) & 12567 & 11261 \pm 334 & 0.90 \\ & \mathrm{G}^{3}(4 \mathrm{~d}, 5 \mathrm{p}) & 12260 & 12260(\mathrm{FIX}) & 1.00 \\ 4 \mathrm{~d}^{9} 5 \mathrm{~s}^{2} 4 \mathrm{f} & \mathrm{E}_{\text {av }} & 684316 & 659204 \pm 284 & 0.96\end{array}$

Configuration Interaction Integrals

$\begin{array}{lllll}5 \mathrm{~s} 5 \mathrm{p}-5 \mathrm{p} 5 \mathrm{~d} & \mathrm{R}^{1}(5 \mathrm{~s} 5 \mathrm{p}, 5 \mathrm{p} 5 \mathrm{~d}) & 65548 & 49167 \text { (FIX) } & 0.75 \\ 5 \mathrm{~s} 5 \mathrm{p}-5 \mathrm{p} 5 \mathrm{~d} & \mathrm{R}^{2}(5 \mathrm{p} 5 \mathrm{~d}, 5 \mathrm{~s} 5 \mathrm{p}) & 48216 & 36167 \text { (FIX) } & 0.75 \\ 5 \mathrm{~s} 5 \mathrm{f}-5 \mathrm{p} 5 \mathrm{~d} & \mathrm{R}^{1}(5 \mathrm{~s} 5 \mathrm{f}, 5 \mathrm{p} 5 \mathrm{~d}) & 28688 & 28688 \text { (FIX) } & 1.00\end{array}$

${ }^{a}$ Parameters omitted from this table: direct and exchange integrals, and spin-orbit $\zeta$ parameters set to $85 \%$ and $95 \%$ of their HFR values respectively; CI integrals were set to $85 \%$ of their HFR values. The standard deviation for the energy adjustment was 281 $\mathrm{cm}^{-1}$.

Table 5 Energy parameters $\left(\mathrm{cm}^{-1}\right)$ for the studied even parity configurations of Xe VIII

\begin{tabular}{|c|c|c|c|c|}
\hline Configuration & \multirow[t]{2}{*}{ Parameter } & \multirow{2}{*}{$\begin{array}{l}\text { H-F value } \\
0\end{array}$} & \multirow{2}{*}{\begin{tabular}{|c|} 
Fitted value \\
$1604 \pm 221$
\end{tabular}} & \multirow[t]{2}{*}{ Fitt/H-F ${ }^{a}$} \\
\hline $5 s$ & & & & \\
\hline $6 s$ & $\mathrm{E}_{\mathrm{av}}$ & 390824 & $395674 \pm 220$ & 1.01 \\
\hline $7 s$ & $\mathrm{E}_{\mathrm{av}}$ & 559648 & $565446 \pm 220$ & 1.01 \\
\hline $8 s$ & $\mathrm{E}_{\mathrm{av}}$ & 648997 & $655344 \pm 221$ & 1.01 \\
\hline $9 \mathrm{~s}$ & $\mathrm{E}_{\mathrm{av}}$ & 702240 & $708802 \pm 221$ & 1.01 \\
\hline $10 \mathrm{~s}$ & $\mathrm{E}_{\mathrm{av}}$ & 738183 & 738183(FIX) & 1.00 \\
\hline $5 d$ & $\mathrm{E}_{\mathrm{av}}$ & 307328 & $311934 \pm 159$ & 1.01 \\
\hline & $\zeta_{5 \mathrm{~d}}$ & 973 & $1166 \pm 125$ & 1.20 \\
\hline
\end{tabular}




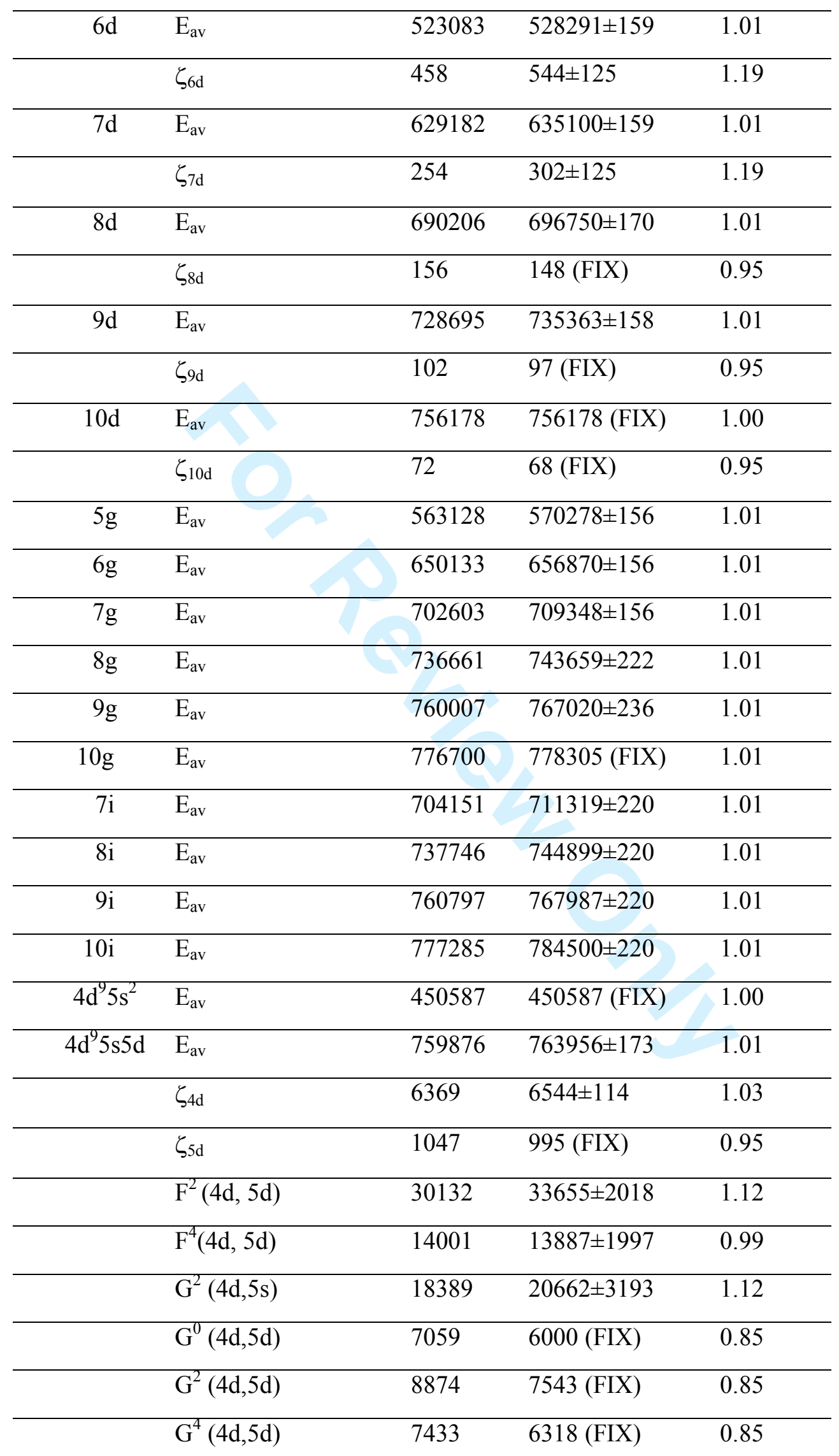




$\begin{array}{lllll} & \mathrm{G}^{2}(5 \mathrm{~s}, 5 \mathrm{~d}) & 42987 & 33247 \pm 1470 & 0.77 \\ 4 \mathrm{~d}^{9} 5 \mathrm{p}^{2} & \mathrm{E}_{\mathrm{av}} & 708344 & 708344(\mathrm{FIX}) & \end{array}$

${ }^{a}$ Parameters omitted from this table: direct and exchange integrals, and spin-orbit $\zeta$ parameters set to $85 \%$ and $95 \%$ of their HFR values respectively; CI integrals were set to $85 \%$ of their HFR values. The standard deviation for the energy adjustment was 220 $\mathrm{cm}^{-1}$.

Table 6 Energy parameters $\left(\mathrm{cm}^{-1}\right)$ for the studied odd parity configurations of Xe VIII

\begin{tabular}{|c|c|c|c|c|}
\hline Configuration & Parameter & H-F value & Fitted value & Fitt/H-F \\
\hline $5 p$ & $\mathrm{E}_{\mathrm{av}}$ & 127266 & $133349 \pm 186$ & 1.05 \\
\hline & $\zeta_{5 p}$ & 11381 & $12447 \pm 235$ & 1.09 \\
\hline $6 p$ & $\mathrm{E}_{\mathrm{av}}$ & 443669 & $449194 \pm 185$ & 1.01 \\
\hline & $\zeta_{6 \mathrm{p}}$ & 4650 & $4933 \pm 235$ & 1.06 \\
\hline $7 p$ & $\mathrm{E}_{\mathrm{av}}$ & 586793 & $593045 \pm 179$ & 1.01 \\
\hline & $\zeta_{7 \mathrm{p}}$ & 2395 & 2275 (FIX) & 0.95 \\
\hline $8 p$ & $\mathrm{E}_{\mathrm{av}}$ & 664825 & $671726 \pm 176$ & 1.01 \\
\hline & $\zeta_{8 \mathrm{p}}$ & 1403 & 1333 (FIX) & 0.95 \\
\hline $9 p$ & $\mathrm{E}_{\mathrm{av}}$ & 712278 & $719104 \pm 186$ & 1.01 \\
\hline & $\zeta_{9 \mathrm{p}}$ & 894 & $922 \pm 235$ & 1.03 \\
\hline $10 p$ & $\mathrm{E}_{\mathrm{av}}$ & 743345 & 743345 (FIX) & 1.00 \\
\hline $4 \mathrm{f}$ & $\mathrm{E}_{\mathrm{av}}$ & 263400 & $265561 \pm 176$ & 1.01 \\
\hline & $\zeta_{4 \mathrm{f}}$ & 282 & 268 (FIX) & 0.95 \\
\hline $5 f$ & $\mathrm{E}_{\mathrm{av}}$ & 493775 & $497866 \pm 175$ & 1.01 \\
\hline & $\zeta_{5 \mathrm{f}}$ & 105 & $100(\mathrm{FIX})$ & 0.95 \\
\hline $6 f$ & $\mathrm{E}_{\mathrm{av}}$ & 611494 & $616577 \pm 176$ & 1.01 \\
\hline & $\zeta_{6 \mathrm{f}}$ & 55 & 52 (FIX) & 0.95 \\
\hline $7 f$ & $\mathrm{E}_{\mathrm{av}}$ & 678916 & $684573 \pm 176$ & 1.01 \\
\hline & $\zeta_{7 f}$ & 33 & 31 (FIX) & 0.95 \\
\hline $8 f$ & $\mathrm{E}_{\mathrm{av}}$ & 721106 & $727379 \pm 175$ & 1.01 \\
\hline & $\zeta_{8 \mathrm{f}}$ & 21 & 20 (FIX) & 0.95 \\
\hline
\end{tabular}




\begin{tabular}{|c|c|c|c|c|}
\hline $9 f$ & $\mathrm{E}_{\mathrm{av}}$ & 749252 & $755759 \pm 175$ & 1.01 \\
\hline & $\zeta_{9 f}$ & 15 & 14 (FIX) & 0.95 \\
\hline $10 \mathrm{f}$ & $\mathrm{E}_{\mathrm{av}}$ & 768949 & 768949 (FIX) & 1.00 \\
\hline $6 \mathrm{~h}$ & $\mathrm{E}_{\mathrm{av}}$ & 651951 & $659192 \pm 176$ & 1.01 \\
\hline $7 \mathrm{~h}$ & $\mathrm{E}_{\mathrm{av}}$ & 703834 & $711059 \pm 248$ & 1.01 \\
\hline $8 \mathrm{~h}$ & $\mathrm{E}_{\mathrm{av}}$ & 737538 & $744772 \pm 248$ & 1.01 \\
\hline $9 \mathrm{~h}$ & $\mathrm{E}_{\mathrm{av}}$ & 760653 & $767888 \pm 248$ & 1.01 \\
\hline $10 \mathrm{~h}$ & $\mathrm{E}_{\mathrm{av}}$ & 777184 & 777184 (FIX) & 1.00 \\
\hline $8 \mathrm{k}$ & $\mathrm{E}_{\mathrm{av}}$ & 737816 & $744951 \pm 248$ & 1.01 \\
\hline $9 \mathrm{k}$ & $\mathrm{E}_{\mathrm{av}}$ & 760848 & $767985 \pm 248$ & 1.01 \\
\hline $10 \mathrm{k}$ & $\mathrm{E}_{\mathrm{av}}$ & 777325 & $784478 \pm 248$ & 1.01 \\
\hline $4 d^{9} 5 s 5 p$ & $\mathrm{E}_{\mathrm{av}}$ & 566334 & $567120 \pm 162$ & 1.01 \\
\hline & $\zeta_{4 d}$ & 6342 & 6025 (FIX) & 0.95 \\
\hline & $\zeta_{5 p}$ & 12229 & $14224 \pm 280$ & 1.16 \\
\hline & $\mathrm{F}^{2}(4 \mathrm{~d}, 5 \mathrm{p})$ & 43498 & $35529 \pm 2304$ & 0.82 \\
\hline & $\mathrm{G}^{2}(4 \mathrm{~d}, 5 \mathrm{~s})$ & 18331 & $19275 \pm 1771$ & 1.05 \\
\hline & $G^{1}(4 d, 5 p)$ & 13040 & $11665 \pm 479$ & 0.89 \\
\hline & $G^{3}(4 d, 5 p)$ & 12788 & 10870 (FIX) & 0.85 \\
\hline & $G^{1}(5 s, 5 p)$ & 80420 & $56775 \pm 702$ & 0.71 \\
\hline $4 d^{9} 4 f 5 s$ & $\mathrm{E}_{\mathrm{av}}$ & 693106 & $651800 \pm 180$ & 0.94 \\
\hline $4 d^{9} 5 p 5 d$ & $\mathrm{E}_{\mathrm{av}}$ & 891877 & 891877 (FIX) & 1.00 \\
\hline
\end{tabular}

${ }^{a}$ Parameters omitted from this table: direct and exchange integrals, and spin-orbit $\zeta$ parameters set to $85 \%$ and $95 \%$ of their HFR values respectively; CI integrals were set to $85 \%$ of their HFR values. The standard deviation for the energy adjustment was 248 $\mathrm{cm}^{-1}$. 